\title{
Helicobacter pylori infection - update for the clinician 2004
}

Auch im Jahr 2004 bleibt die Beschäftigung mit der Helicobacter-pylori(H.p.)-Infektion eine spannende Herausforderung für Klinik und Grundlagenforschung (46). Die H.p.-Therapie zur Heilung der peptischen Ulkuskrankheit ist zwar allgemein akzeptiert, allerdings lässt die letzte Konsequenz der Umsetzung dieser Behandlung in der Praxis immer noch Wünsche offen. Die klinische Diskussion dreht sich weiterhin um Indikationsstellungen zur Behandlung der H.p.-Infektion, wenn noch keine Komplikationen eingetreten sind. Aktuell sind vor allem Fragen, ob Patienten mit dyspeptischen Beschwerden bei H.p.-positiver Gastritis von dieser Therapie profitieren, ob die Behandlung der Infektion vor Schäden durch nicht-steroidale Antirheumatika (NSAR) schützt, und nicht zuletzt, ob eine Prävention des Magenkarzinoms durch H.p.-Eradikation erreicht werden kann.

\section{Epidemiologie und Übertragungswege}

Die Prävalenz der H.p.-Infektion in verschiedenen Populationen weist große regionale Unterschiede auf. In Europa ist die Durchseuchung in den Ländern des Mittelmeerraumes und des Ostens am höchsten. So sind in Ländern wie Rumänien oder der Türkei bereits Kinder zu 60\% und mehr mit H.p. infiziert. Noch gilt, dass mit zunehmendem Alter die Durchseuchungsprävalenz steigt, d.h. pro Lebensjahr ist ein Anstieg der H.p.-Prävalenz um 0,3-1\% zu erwarten, in Entwicklungsländern sogar um 1,9\%. In den Ländern der westlichen Welt ist in Zukunft von einem Prävalenzrückgang um 10-25\% im Erwachsenenalter auszugehen (44). In aktuellen Untersuchungen wird gezeigt, dass die H.p.Prävalenz bei Jugendlichen zwischen dem 16. und 20. Lebensjahr in der Schweiz bei 7,3\% liegt, in Deutschland noch bei 9,4\% (16, 17, 44), genauer gesagt bei $7,1 \%$ bei deutschen Jugendlichen und bei $28,2 \%$ bei Jugendlichen anderer Herkunft.

Die meisten Infektionen mit H.p. erfolgen im Kindes- und Jugendalter; dabei besteht eine starke Abhängigkeit vom sozio-ökonomischen Status $(15,42)$. Der höchste Prävalenzanstieg wird vom 2 . ins 3. Lebensjahr (10\% versus 32\%) gefunden (14). Bei Erwachsenen zeigte eine epidemiologische Studie aus Brasilien einen Anstieg der H.p.Seroprävalenz von 23,4\% bei Studenten zu Beginn des Medizinstudi- ums auf 38,6\% bei Ärzten im Praktikum (37). Zu ähnlichen Ergebnissen kommt eine Arbeit aus Griechenland, wo medizinisches und nichtmedizinisches Personal in Krankenhäusern im 5-Jahres-Verlauf untersucht wurde. Die initiale Seroprävalenz lag bei $45,5 \%$. Nach 5 Jahren war bei $24,8 \%$ der zuvor negativen Personen eine Serokonversion zu verzeichnen. Diese war beim medizinischen signifikant höher als beim nicht-medizinischen Personal (47).

Da der Magen des Menschen das Hauptreservoir für H.p. darstellt, kann die Infektion entweder oral-oral, fäkal-oral oder gastro-oral übertragen werden. Bisher gelang es nur in Einzelfällen, H.p. aus dem Speichel, aus Zahnplaque oder aus dem Stuhl anzuzüchten (9). Auch die PCR als Nachweismethode brachte keinen entscheidenden Fortschritt. Im Gegensatz dazu lassen sich mit Hilfe der ELISA-Technik spezifische H.p.-Antigene im Stuhl feststellen (26), und diese Methode könnte zur weiteren Klärung des fäkal-oralen Transmissionsweges künftig beitragen. Als weiterer Übertragungsmodus wird die gastro-orale Route diskutiert. So gibt es Untersuchungen, dass die Übertragung durch den Magensaft, speziell als Ergebnis endemischen Erbrechens im Rahmen von Virusinfektionen oder durch gastroösophagealen Reflux erfolgen kann.

Eltern und Geschwister H.p.-positiver Kinder sind in einem viel höheren Maße ebenfalls H.p.-positiv als die Familienangehörigen von H.p.-negativen Kindern (43). Die Übertragung bei Ehepartnern erscheint eher unwahrscheinlich. Nach erfolgreicher Eradikationstherapie im Erwachsenenalter bleibt die Zahl der echten Re-Infektionen sehr gering (38).

lkurzgefasst: Die H.p.-Infektion zeigt weltweit eine sinkende Prävalenz. Sie wird im frühen Kindesalter erworben, das Neuinfektionsrisiko des Erwachsenen ist gering. Eine Ausnahme stellen Risiko-Berufsgruppen dar. In Ländern mit gehobenem Hygienestandard kommt in erster Linie die oral-orale Übertragung in Betracht, unter schlechten hygienischen Bedingungen wie in Entwicklungsländern auch der fäkal-orale Infektionsweg.

Prof. Dr. P. Malfertheiner · Klinik für Gastroenterologie, Hepatologie und Infektiologie der Otto-von-GuerickeUniversität Magdeburg · Leipziger Str. $44 \cdot 39120$ Magdeburg · Tel.: 0391-6713100 · Fax: 0391-6713105 . E-Mail: peter.malfertheiner@medizin.uni-magdeburg.de 
Tab.1 Empfohlene Methoden zur H.p.-Diagnostik, abhängig von der klinischen Problemstellung.

\begin{tabular}{|c|c|c|}
\hline Problemstellung & & Test \\
\hline \multicolumn{3}{|l|}{ Prätherapeutisch } \\
\hline \multirow[t]{2}{*}{$\begin{array}{l}\text { Routinesituation } \\
\text { (dyspeptische } \\
\text { Beschwerden) }\end{array}$} & $\begin{array}{l}\text { nicht-invasive Diagnostik aus- } \\
\text { reichend (Dyspepsie, } \\
\text { Alter<45 Jahre, keine Alarm- } \\
\text { symptome, keine NSAR, keine } \\
\text { Familienvorgeschichte für } \\
\text { Magenkarzinom) }\end{array}$ & $\begin{array}{l}\text { Harnstoff-Atemtest } \\
\text { oder H.p.-Antigen- } \\
\text { Stuhltest }\end{array}$ \\
\hline & endoskopisch & $\begin{array}{l}\text { HUT, wenn negativ auch } \\
\text { Histologie }\end{array}$ \\
\hline \multirow[t]{4}{*}{ Problemsituation } & $\begin{array}{l}\text { Vorbehandlung mit H.p.-sup- } \\
\text { primierender Therapie (Proto- } \\
\text { nenpumpenhemmer, } \\
\text { Antibiotika) }\end{array}$ & $\begin{array}{l}\text { mindestens } 2 \text { Wochen } \\
\text { vorher absetzen! }\end{array}$ \\
\hline & Blutung aus peptischem Ulkus & $\begin{array}{l}\text { wenn möglich HUT und } \\
\text { Histologie vor Beginn } \\
\text { einer H.p.-supprimie- } \\
\text { renden Therapie }\end{array}$ \\
\hline & $\begin{array}{l}\text { nicht korrigierbare Gerin- } \\
\text { nungsstörung }\end{array}$ & $\begin{array}{l}\text { Harnstoff-Atemtestund } \\
\text { H.p.-Antigen-Stuhltest, } \\
\text { falls nicht möglich H.p.- } \\
\text { IgG-Serologie }\end{array}$ \\
\hline & Magenresektion & $\begin{array}{l}\text { HUT und Histologie } \\
\text { aus Magenfundus }\end{array}$ \\
\hline \multicolumn{3}{|l|}{ Posttherapeutisch } \\
\hline & $\begin{array}{l}\text { Symptomfreiheit bei unkomp- } \\
\text { liziertem Ulcus duodeni oder } \\
\text { funktioneller Dyspepsie }\end{array}$ & Harnstoff-Atemtest \\
\hline & $\begin{array}{l}\text { Beschwerdepersistenz oder } \\
\text { Ulcus ventriculi oder } \\
\text { MALT-Lymphom }\end{array}$ & $\begin{array}{l}\text { HUT, Histologie, Kultur } \\
\text { mit Resistenzbestim- } \\
\text { mung }\end{array}$ \\
\hline \multicolumn{3}{|c|}{ HUT: Helicobacter-Urease-Schnelltest } \\
\hline
\end{tabular}

\section{Diagnostik}

Neuentwicklungen haben sich insbesondere bei nicht-invasiven Tests und zur H.p.-Diagnostik in Problemsituationen ergeben (Tab.1). Für die klinische Praxis ist neben der Einteilung in nicht-invasive $\left({ }^{13} \mathrm{C}\right.$-Harnstoff-Atemtest, Stuhlantigentest, Antikörper-Tests) und invasive Tests (Ureaseschnelltest, Histologie, Kultur) die Unterteilung in direkte und indirekte Tests bedeutsam. Mit den direkten Tests werden Bakterien (Kultur, Histologie), Bakterienbestandteile (Stuhlantigentest) oder ihre Stoffwechselprodukte (Ureaseschnelltest, ${ }^{13} \mathrm{C}$-Harnstoff-Atemtest) nachgewiesen. Mit indirekten Tests sind die Antikörpernachweismethoden gemeint. Der Vorteil der direkten Methoden liegt darin, dass eine aktuelle Infektion nachgewiesen wird, während der Antikörpernachweis auch positiv ausfallen kann, wenn die Infektion schon Monate oder Jahre eliminiert ist. Nachteil der direkten Tests ist, dass sie im Gegensatz zu Antikörpertests von der Kolonisationsdichte abhängig sind. Beeinträchtigt wird diese insbesondere von H.p. supprimierenden Medikamenten, wie Antibiotika und Protonenpumpenhemmer (PPI), aber auch von einer verminderten Säureproduktion, wie sie bei korpusdominanter Gastritis oder Magenschleimhautatrophie angetroffen wird.

Nachteile der Antikörper-Tests sind falsch-positive Befunde aufgrund möglicher Kreuzreaktionen und falsch-negative Befunde durch fehlende Antigenität oder fehlende Immunantwort. Eine weitere Ursache hierfür ist die hohe genetische Variabilität von H.p., die sich in interindividuellen und insbesondere geographischen Unterschieden der relevanten H.p.-Epitope niederschlägt. Daher müssen serologische Methoden regional validiert werden.

\section{Nicht-invasive Diagnostik und H.p.-Schnelltests}

Alle bisher entwickelten laborunabhängigen Schnelltests beruhen auf immunologischen Tests. Bislang hat keiner dieser Tests im Blut, im Urin oder im Speichel die klinisch notwendige diagnostische $\mathrm{Zu}-$ verlässigkeit zur Empfehlung für die klinische Praxis erreicht (25, 26, 28,29 ). Vielversprechender dagegen sind immunologische Tests, die H.p.-Antigene im Stuhl nachweisen. Zwei kommerziell erhältliche Stuhlantigentests haben eine ausreichende Sensitivität und Spezifität, sind jedoch auf ein Labor mit ELISA-Technik angewiesen. Erste Ergebnisse eines laborunabhängigen Stuhlantigen-Schnelltests haben ergeben, dass die Sensitivität nicht an die der laborgestützten ELISA-Tests heranreicht (29). Somit kann der Stuhlantigenschnelltest im derzeitigen Entwicklungsstand noch nicht für die Praxis empfohlen werden. Diagnostischer Standard der nicht-invasiven Diagnostik sind daher weiterhin der ${ }^{13} \mathrm{C}$-Harnstoff-Atemtest und der Stuhlantigentest $(24,27)$.

\section{Diagnostik beim Spezialisten}

Die invasive Routinediagnostik beruht auf endoskopisch entnommenen Biopsien für den Urease-Schnelltest und die Histologie. Wegen des individuell sehr unterschiedlichen Säuresekretionsverhaltens und der daraus resultierenden unterschiedlichen Kolonisationsdichte empfiehlt es sich, immer Biopsien sowohl aus dem Magenantrum, ca. $3-5 \mathrm{~cm}$ präpylorisch und aus dem Magenkorpus, oberes Korpusdrittel, zu entnehmen (je 1 Biopsie für HUT, je 2 für Histologie) (25). Fällt der Urease-Schnelltest positiv aus, ist eine Histologie nicht zwingend notwendig, es sei denn, die Biopsien wurden aus endoskopisch suspekten Läsionen entnommen, die einer weiteren histologischen Beurteilung bedürfen. Bei negativem UreaseSchnelltest empfiehlt es sich, die Histologie dem Pathologen einzusenden, da die Histologie noch einmal eine höhere Sensitivität als der Urease-Schnelltest hat. Außerdem erhält man Informationen über die Aktivität der Gastritis oder über eventuelle Sonderformen von Gastritis.

\section{Problemsituationen}

Zustand nach Magenteilresektion. Verschiedene H.p.-Tests wurden bei Z.n. Billroth-I- oder -II-Resektion validiert. Da diese Zustände mit einer verminderten Säuresekretion einhergehen und zudem häufig mit einem Gallereflux verbunden sind, zeigte sich hier erwartungsgemäß eine deutlich verminderte Sensitivität der Tests. Dennoch ist die H.p.-Diagnostik in einer solchen Situation von Bedeutung, wenn es zu rezidivierenden Ulzera, meist im Bereich der Anastomose kommt. Aufgrund der verminderten Säuresekretion empfiehlt es sich, Biopsien aus dem Korpus und insbesondere aus dem Fundus zu entnehmen, da hier noch das Maximum der verbliebenen Säuresekretion stattfindet. In jedem Fall sollte der Urease-Schnelltest durch eine histologische Untersuchung ergänzt werden. Ein Harnstoff-Atemtest erlaubt in dieser Situation keine verlässliche Aussage.

Peptische Ulkusblutung und Gerinnungsstörung. Bei der peptischen Ulkusblutung ist eine exakte H.p.-Diagnostik von besonderer Bedeutung, da durch Beseitigung der Infektion, die Rezidivrate der Ulkusblutung auch im Vergleich mit einer säuresupprimierenden 
Dauertherapie deutlich gesenkt werden kann. Es empfiehlt sich daher, bereits bei der initialen Endoskopie Biopsien für einen UreaseSchnelltest und die Histologie zu entnehmen. Eine Reihe von Studien hat gezeigt, dass der Urease-Schnelltest deutlich weniger sensitiv ist, wenn sich Blut im Magen findet (45). Wir konnten dies nicht bestätigen, fanden jedoch bei Patienten mit einer peptischen Ulkusblutung eine rasche Abnahme der Sensitivität aller direkten Methoden innerhalb weniger Tage nach Beginn der H.p.-supprimierenden Therapie (41). Bei Vorbehandlung mit H.p.-supprimierender Therapie sollten auch Biopsien aus dem Magenfundus entnommen werden.

Ein Teil der Patienten mit peptischer Ulkusblutung steht unter Antikoagulation, z.B. nach kardiovaskulären Eingriffen. In einem solchen Fall ist man auf nicht-invasive Tests angewiesen. Serologische Tests sind nicht ausreichend sensitiv und spezifisch. Es empfiehlt sich, einen Harnstoff-Atemtest oder einen Antigen-Stuhltest möglichst noch am Tag der Diagnose der peptischen Ulkusblutung durchzuführen. Auch der H.p.-Antigen-Stuhltest ist ab etwa dem 3. Tag nach einer Therapie mit H.p.-supprimierenden Medikamenten nicht ausreichend sensitiv.

\section{Posttherapeutische Kontrolle}

Wenn eine Pause aller H.p.-supprimierenden Medikamente von 4 Wochen gewährleistet ist, sind alle direkten Test in gleicher Weise wie in der prätherapeutischen Situation auch für die Therapiekontrolle geeignet. In den meisten Fällen kann eine Therapiekontrolle entweder mit dem Atem- oder Stuhltest erfolgen (31). Die Notwendigkeit zur Endoskopie ergibt sich aus weiteren Gesichtspunkten, wie dem Malignitätsausschluss beim Magenulkus oder zur Überprüfung der Ulkusheilung nach einer Blutung.

\section{lkurzgefasst: Von Problemsituationen abgesehen wird eine bioptische Diagnostik immer durchgeführt, wenn eine Endoskopie indiziert ist. Die nicht-invasive Diag- nostik (Atemtest, Stuhltest) ist erste Wahl zur Eradikati- onskontrolle. H.p.-supprimierende Medikamente soll- ten 2 Wochen vor elektiver Testung abgesetzt werden. Die Kontrolle des Eradikationserfolges kann frühestens 4 Wochen nach Absetzen solcher Medikamente erfolgen.}

\section{Indikationen zur H.p.-Therapie}

Seit dem Jahr 2000 liegt eine Liste von Therapieempfehlungen aus der europäischen H.p.-Konferenz in Maastricht vor, die in strenge, ratsame und ungeklärte Indikationskategorien unterteilt werden (31) (Tab.2). Neben der Standardindikation der peptischen Ulkuskrankheit und einer Reihe weiterer bereits eingetretener Komplikationen der H.p.-Infektion gilt insbesondere die Behandlung des MALT-Lymphoms des Magens als wichtige Indikation (39). Dies umso mehr, als auch im Langzeitverlauf das exzellente Ergebnis der vollständigen Remission dieser Neoplasie bestätigt wurde (11). Inzwischen gilt als gesichert, dass vor erstmaliger Gabe von NSAR und Acetylsalicylsäure (ASS) die H.p.-Eradikation in der Regel einen guten Schutz vor Magen- und Zwölffingerdarmläsionen bietet (5, 22). Ein ausgeprägter Einfluss der H.p.-Eradikation auf den Heilungsverlauf unter gleichzeitiger PPI-Therapie ist nicht gegeben. Nach gastrointestinalen Blutungen ist bei Weiterführen der NSAR-Therapie die H.p.-Eradikation indiziert, allerdings bleibt zusätzlich eine dauerhafte PPI-Therapie erforderlich. Kontrovers ist die Datenlage bei gastrointestinalen Blutungen als Folge einer ASS-Einnahme. Hierzu gibt es zwei große Studien, beide aus Hongkong, von denen die eine einen
Tab. 2 H.p.-Infektion: Wen behandeln?

a) Streng empfohlen

Wissenschaftliche Evidenz

Doudenalulkus/Magenulkus (aktiv oder nicht, einschließ- 1 lich der komplizierten Ulzera)

Malt-Lymphom (niedrig maligne)

1

Atrophische Gastritis

2

Z. n. partieller Magenresektion (bei Magenkarzinom, pep- 3 tischem Ulkus)

Verwandte 1. Grades von Patienten mit Magenkrebs Patientenwunsch

b) Ratsame Empfehlungen - relevante klinische Aussagen

\section{Funktionelle Dyspepsie}

H.p.-Eradikation ist eine adäquate therapeutische Option 2

Die Behandlung der H.p.-Infektion führt zu einer anhal- 2 tenden Verbesserung der Beschwerden bei einer begrenzten Gruppe von Patienten (ca. 10\% besser im Vergleich zur symptomatischen Therapie)

\section{Gastroösophageale Refluxkrankheit (GERD)}

H.p.-Eradikation

...führt in den allermeisten Fällen nicht zu einer Neuentwicklung der GERD

...führt nicht zu einer Verschlimmerung der GERD 3

Die H.p.-Behandlung sollte bei Patienten erfolgen, die eine 3 Langzeitbehandlung mit einem potenten Säuresekretionshemmer bekommen

\section{Nicht-steroidale Antirheumatika}

H.p. und NSAR / ASS sind unabhängige Risikofaktoren für 2 die peptische Ulkuskrankheit

Die Behandlung der H.p.-Infektion

...führt zu einer Reduktion von Ulzera, wenn sie vor dem Gebrauch von NSAR erfolgt.

...allein ist nicht ausreichend, um rezidivierende Ulkusblu- 2 tungen bei einer Hochrisikogruppe von NSAR-Verbrauchern zu vermeiden.

...führt nichtzu einer rascheren Heilung bei Pat. mit Magen- 1 und Duodenalulkus, die NSAR weiterhin nehmen.

1= höchster Evidenzgrad; 4=niedrigster Evidenzgrad

ausreichenden Schutz durch alleinige H.p.-Eradikation nachweist (4), während die andere Studie dies nicht bestätigt und nach Eradikation die Weiterführung der PPI-Therapie empfiehlt (23).

Bei Patienten, bei denen nach Ausschluss organischer Ursachen endoskopisch eine H.p.-positive funktionelle Dyspepsie diagnostiziert wurde, war in einer großen multizentrischen Studie in Deutschland die Eradikationstherapie zur Reduktion der Beschwerden effektiver als die PPI Therapie in einem Nachbeobachtungszeitraum von einem Jahr (32).

\section{H.p.-Therapie in der Praxis: das Problem der Dyspepsie}

Für die Behandlung von Patienten mit dyspeptischen Beschwerden in der Praxis liegen große Studien vor, die zeigen, dass bei unkomplizierter Dyspepsie (ohne Vorliegen von Alarmsymptomen) die nicht-invasive Testung auf eine H.p.-Infektion mit anschließender Behandlung effektiv und sicher ist $(6,35)$. Eine dieser Studien zeigt, dass diese Strategie von den Patienten sogar bevorzugt wird (35) und eine weitere Studie belegt einen günstigen Kosten-Nutzen-Effekt dafür (6). Die H.p.-Eradikation bei H.p.-infizierten Patienten ist der empirischen Therapie mit PPI bei längerer Nachbeobachtung überlegen $(7,34)$. Grund für diesen Therapievorteil mittels „Test- and Treat“-Strategie ist, dass 
sich auch Patienten mit Ulkusleiden darunter befinden, die in jedem Falle durch die H.p.-Therapie geheilt wurden. Auch Patienten mit H.p.induzierter funktioneller Dyspepsie befinden sich darunter und können zumindest zu einem Teil von den Beschwerden befreit werden. In Deutschland lässt sich diese Strategie nach wie vor nur schwer durchsetzen, da insbesondere aufgrund der geringen Kosten der Endoskopie die Test-and-Treat-Strategie gegenüber der primären Endoskopie-basierten Strategie in Deutschland nicht von Vorteil erscheint (1).

\section{H.p. und gastroösophageale Refluxkrankheit: eine andauernde Kontroverse}

Der Zusammenhang der H.p.-Infektion und gastroösophagealen Refluxkrankheit (GERD) wird kontrovers beurteilt; sie wird von manchen Autoren sogar als eine Warnung gegen den zu „großzügigen“ Einsatz der Eradikationstherapie herangezogen. Dass zwischen der abnehmenden H.p.-Prävalenz und der steigenden Prävalenz der Refluxkrankheit in der Bevölkerung entwickelter Länder ein kausaler Zusammenhang besteht (2), ist unseres Erachtens eine Überinterpretiation, da sich zahlreiche andere Lebensbedingungen in dieser Zeit ebenfalls verändert haben. Die Prävalenz der H.p.-Infektion bei Patienten mit GERD liegt in Deutschland bei etwa 25\%. Der Verlauf der GERD wird durch Eradikation der H.p.-Infektion nicht signifikant beeinflusst (21). Die Beobachtung, dass nach Beseitigung der Infektion die Wirkung der PPI auch bei Dauertherapie abnimmt, ist von geringer klinischer Bedeutung und gilt nur bei Anwendung suboptimaler PPI-Dosierungen.

Kontrovers ist die Datenlage, ob eine Eradikation der H.p.-Infektion zu einem Neuauftreten der GERD führen kann. Unsere Einschätzung dieses Sachverhaltes nach einer Reihe neuerer Publikationen der letzten beiden Jahre ist, dass, wenn überhaupt, nur bei ausgewählten Patienten mit entsprechender Prädisposition nach H.p.-Eradikation das Auftreten der GERD möglich wird. Voraussetzung dafür ist eine prädominante atrophische Korpusgastritis mit verminderter Säuresekretion, die nach H.p.-Eradikation reversibel ist und auf eine gestörte gastroösophageale Refluxbarriere trifft (30).

Die überwiegende Zahl aktueller Studiendaten lässt ein erhöhtes Risiko für eine Refluxkrankheit bzw. für das (Wieder-) Auftreten von Refluxsymptomen nach erfolgreicher H.p.-Eradikation nicht erkennen. Retrospektive (30) Analysen multizentrischer Studien bei Patienten mit peptischen Ulzera zeigen, dass die erfolgreiche Eradikation von H.p. im Nachbeobachtungszeitraum von 6 Monaten eher zu einer Abnahme der Prävalenz von Refluxsymptomen führt. Das Auftreten der Refluxösophagitis im zeitlichen Verlauf war unabhängig vom H.p.-Status nach Eradikation. Eine Erklärung für die beobachteten Differenzen in den Studienergebnissen könnte auch die Demaskierung präexistenter GERD-Symptome nach erfolgreicher Eradikation und Beseitigung der Ulkusschmerzen sein.

klurzgefasst: Zurückhaltung in der Anwendung einer H.p.Therapie wegen einer „Ex-novo“-GERD erscheint nicht angemessen. Eine Verschlechterung von GERD durch die H.p.-Eradikation ist nicht zu erwarten. Die H.p.-Therapie einer funktionellen Dyspepsie sollte aufgrund des günstigen Kosten-Nutzen-Profils vor Langzeittherapie mit anderen Medikamenten erwogen werden. Schleimhautläsionen durch ASS/NSAR in der Primär-/ und Sekundärprophylaxe werden in vielen Fällen durch die Eradikation günstig beeinflusst. Nach Blutungskomplikationen ist trotz H.p.-Eradikation eine Langzeit-PPI-Gabe erforderlich.
Tab. 3 Therapieübersicht.

\section{Maastricht - II: Primärtherapie}

PPI Standarddosis bid + Clarithromycin $500 \mathrm{mg}(\mathrm{C})^{*}+$ Amoxicillin $1 \mathrm{~g}(\mathrm{~A})$ oder Metronidazol $400 \mathrm{mg}(\mathrm{M})^{*}$, alle Medikamente zweimal täglich für $\geq 7$ Tage ${ }^{*} \mathrm{CA}$ ist CM vorzuziehen, da geringere Resistenzprobleme $\downarrow$

\section{Sekundärtherapie}

$\downarrow$

PPI $2 \times / d+$ Wismutsubcitrat $120 \mathrm{mg} 4 \times / d+$ Metronidazol $400 \mathrm{mg} 3 \times / d$ + Tetracyclin $500 \mathrm{mg} 4 \times /$ f für $\geq 7$ Tage

Falls kein Wismut verfügbar bzw. kontraindiziert sollte eine adäquate PPIgestützte Therapie zur Anwendung kommen

Bei fortgesetztem Therapieversagen: Individuelles Management (resistenzgerecht) (s. Tab.4)

\section{Therapie der H.p.-Infektion}

Seit Einführung der Tripeltherapie als Standard-Eradikationsbehandlung liegt der Behandlungserfolg bei etwa 90\% (31). Die wichtigsten Gründe für ein Therapieversagen liegen in der ungenügenden Compliance der Patienten und Antibiotikaresistenz sowie im Auftreten von unerwünschten Arzneimittelwirkungen, die zum vorzeitigen Therapieabbruch führen. Gründe für die ungenügende Compliance sind unerwünschte Nebenwirkungen und unzureichende Aufklärung der Patienten.

Bei korrekter Einnahme der Medikamente ist die Resistenz gegenüber den eingesetzten Antibiotika von Bedeutung $(19,31,49)$. Für Clarithromycin als Standardsubstanz besteht eine eindeutige Wirkungsbeziehung zum Therapieversagen, wenn eine Resistenz vorliegt. Bei Imidazolen hingegen ist die mikrobiologische Resistenz nicht in jedem Fall mit klinischer Resistenz gleichzusetzen, da sie zum Teil durch eine Dosiserhöhung oder eine resistenzmodulierende Komedikation wie Amoxicillin bzw. Wismutsalze vermindert wird. Eine wichtige Nebenwirkung von Antibiotika ist die Antibiotika-assoziierte Diarrhoe; diese tritt am häufigsten unter Amoxicillin auf. Daneben sind Geschmacksstörungen klinisch relevant; diese sind überwiegend mit Metronidazol und Makroliden assoziiert. Allergien können prinzipiell mit allen verwendeten Medikamenten beobachtet werden (am häufigsten unter Amoxicillin). Neben der schnelleren Abheilung von Läsionen im Magen und Duodenum besteht die Rationale für die zusätzliche Verwendung eines Säuresekretionshemmers darin, dass ein Teil der verwendeten Antibiotika (Clarithromycin und Amoxicillin) erst durch Anhebung des pH-Werts wirksam werden. Die Säuresuppression führt auch zur Erhöhung der lokalen Konzentrationen dieser Antibiotika, indem das Volumen des produzierten Magensaftes sich verringert. Medikament der Wahl sind Protonenpumpenhemmer in zweimal täglicher Standarddosis (31). Ein neuer Aspekt der Therapie mit PPI wurde kürzlich hinsichtlich unterschiedlicher Metabolisierungsraten aufgezeigt (20).

Aufgrund der umfangreichen Datenlage wird als Erstlinientherapie die Kombination eines Protonenpumpenhemmers und Clarithromycin in Kombination mit Amoxicillin (PPI-C-A) empfohlen (Tab.3) (31). Alternativ kann Amoxicillin durch Metronidazol ersetzt werden (PPIC-M). Beide Regime werden als sogenannte Standard-Triple-Schema für jeweils eine Woche verabreicht. Zwar wäre eine individuelle Resistenz-Testung optimal, für die Praxis reicht jedoch die Kenntnis der 
Tab. 4 Vorgehen bei wiederholtem Therapieversagen.

1. Resistenztestung im Speziallabor (Portagerm-Transportmedium),
resistenzgerechte Therapie*
2. PPI-Amoxicillin Hochdosis für 2 Wochen
3. PPI-Amoxicillin-Rifabutin-(oder Levofloxacin) für 1-2 Wochen
4. PPI-Wismut-Tetracyclin-Furazolidone für 1 Woche
*besonders im Falle der Kombination PPI-Amoxicillin-Clarithromycin sollte
außerhalb der Primärtherapie über 14 Tage behandelt werden!

Resistenzlage in der Region aus $(13,19,31,40)$. Unter diesem Aspekt hat die Kombination mit PPI-C-A Vorteile (18), da eine primäre Resistenz von H.p. gegenüber Metronidazol (bei ca. 30\% der Patienten zu erwarten) keine Rolle spielt. Eine primäre und sekundäre Resistenz gegen Amoxicillin ist nach wie vor die Ausnahme. Für PPI-C-M als zweitbeste Wahl spricht, dass diese Kombination im Falle einer Amoxicillin-Allergie verwendet werden kann und mit geringerem Auftreten von Diarrhoen verbunden ist (26). Falls Nebenwirkungen zu einem vorzeitigen Abbruch führen, geht eine Therapiedauer unter einer Woche bei PPI-C-M seltener mit Therapieversagen einher als bei PPIC-A. Generell sollte nicht kürzer als eine Woche therapiert werden. Der Eradikationserfolg soll durch eine adäquate Diagnostik überprüft werden. Dies kann aufgrund der klinischen Indikation im Rahmen einer Kontroll-Endoskopie mit Biopsieentnahme für Urease-Schnelltest und Histologie (im Idealfall einschließlich mikrobieller Anzüchtung) geschehen. Für die meisten Fälle bietet es sich an, den Therapieerfolg mittels Harnstoff-Atemtest oder Stuhl-Antigentest zu überprüfen.

Bei dokumentiertem Therapieversagen wird eine ebenfalls einwöchige standardisierte Zweitlinientherapie mit PPI-Wismut-Metronidazol-Tetracyclin vorgeschlagen (31). Interessanterweise ist dieses Vierfach-Schema selbst bei Vorhandensein einer Metronidazol-Resistenz nahezu gleich wirksam. Während die standardisierte Erst- und Zweitlinien-Therapie durch den niedergelassenen Allgemeinarzt oder Internisten erfolgen kann, sollte jeder darüber hinausgehende Therapieversuch durch den Gastroenterologen vorgenommen werden, um eine resistenzbasierte individualisierte Therapie einzuleiten (Tab.4).

Wenn aufgrund der Resistenzlage keine Makrolidresistenz vorliegt, kann die Kombination von PPI-A-C durchaus erneut angewandt werden, dann in verlängerter Therapiedauer von 10-14 Tagen. Als Reservetherapie ist in Deutschland die Kombination PPI-Amoxicillin-Rifabutin verfügbar (12), welche ebenfalls für eine Woche gegeben werden kann. Die Option der Hochdosisdualtherapie mit PPI-Amoxicillin, für 2 Wochen stellt eine weitere Option dar; sie ist allerdings häufiger mit Nebenwirkungen wie Diarrhoe belastet (33). Neuere, aber noch nicht ausreichend geprüfte Ansätze sind Triple-Therapien mit PPI Amoxicillin - Levofloxazin oder Moxifloxazin. Die Ergebnisse weiterer klinischer Studien müssen abgewartet werden, bevor diese Kombinationen für den Routinegebrauch bei Therapieversagen empfohlen werden können.

kurzgefasst: Die Ersttherapie der H.p.-Infektion ist nach wie vor ein einwöchiges Tripleschema, bestehend aus je zweimal täglich PPI, Clarithromycin und Amoxicillin, letzteres kann z.B. bei Penicillinallergie durch Metronidazol ersetzt werden. Als Zweitlinientherapie steht eine Vierfachtherapie mit PPI, Wismut, Metronidazol und Tetracyclin zur Verfügung. Weitere Behandlungen nach mehrfachem Eradikationsversagen sollten unter Berücksichtigung der bakteriellen Resistenzbestimmung durch einen Spezialisten erfolgen.

\section{Prophylaxe des Magenkarzinoms durch eine H.p.-Therapie} Seit der Zuordnung der H.p.-Infektion in die Klasse eines Karzinogens durch eine Arbeitsgruppe der WHO 1994 ist der Evidenzgrad für die bedeutsame Rolle der H.p.-Infektion in der Magenkarzinogenese durch viele weitere Befunde angehoben worden. Neuere epidemiologische Untersuchungen zeigen, dass bei Anwendung adäquater Methoden das Risiko von H.p.-Infizierten an einem Magenkarzinom zu erkranken, um das 20-fache erhöht ist. In einer Studie aus Deutschland wird die H.p.-Infektion sogar als Conditio sine qua non für die Magenkarzinomentwicklung aufgezeigt (3). Studien an Tiermodellen und insbesondere zellbiologische Untersuchungen zeigen die direkte Einflussnahme von H.p. auf zelluläre Signalkaskaden, die im Prozess der Karzinogenese beteiligt sind (8). Die Frage, ob eine H.p.-Eradikation auch tatsächlich das Auftreten des Magenkarzinoms verhindert, ist bislang nur in einer großen Beobachtungsstudie aus Japan belegt (48). Eine kürzlich veröffentlichte weitere Arbeit aus China zeigt etwas differenzierter, dass die Karzinomentwicklung durch die H.p.-Eradikation nur dann vermeidbar ist, wenn die Gastritis in ihrer Evolution noch nicht bis zum Grad von atrophischen Veränderungen (Präneoplasie) fortgeschritten ist (50).

Als Konsequenz für die klinische Praxis ist derzeit im Einklang mit den Maastricht-Empfehlungen eine proaktive Rolle bei Familienangehörigen I. Grades von Patienten mit Magenkarzinom angezeigt, des Weiteren bei Patienten mit einer Korpus-dominanten Gastritis $(10,31,36)$. Diese Gruppe mit erhöhtem Magenkarzinomrisiko sollte auf eine H.p.-Infektion untersucht und bei positivem H.p.-Status behandelt werden. Aufgrund des häufigen sporadischen Auftretens des Magenkarzinoms (in Deutschland nach Angaben von Globocan über 19000 Fälle/Jahr) muss an der Entwicklung für eine Screeningund Behandlungsstrategie für die gesamte Bevölkerung kosteneffizienter weiter intensiv gearbeitet werden.

lkurzgefasst: Risikogruppen für die Entwicklung eines Magenkarzinoms sollten systematisch auf eine H.p.-Infektion untersucht und präventiv behandelt werden.

\section{Fazit}

Die H.p.-Infektion stellt nach wie vor die Hauptursache von Magenund Duodenalerkrankungen dar. Für die Diagnostik steht eine Vielzahl direkter und indirekter Nachweisverfahren zur Verfügung, die abhängig von der klinischen Situation differenziert eingesetzt werden können. Der Indikationsbereich für die H.p.-Therapie ist weit gesteckt und der therapeutische Nutzen für die einzelnen Indikationen ist sehr unterschiedlich. Bei manifesten H.p.-induzierten Erkrankungen ist die Behandlung uneingeschränkt indiziert, während die H.p.-Behandlung zur Prävention möglicher Komplikationen kontrovers diskutiert wird. GERD stellt keine Kontraindikation zur Behandlung der H.p. dar. Die Therapie beruht auf dem Konzept der potenten Säurehemmung und Verabreichung von zwei Antibiotika (Triple-Therapie). Bei Therapieversagen stehen eine Reihe alternativer Therapieoptionen zur Verfügung. Bei wiederholtem Therapieversagen ist eine individuelle resistenz-adaptierte Therapie durch den Spezialisten zwingend.

Autorenerklärung: Die Autoren haben wissenschaftliche projektbezogene Verbindungen mit den Firmen Abott, Astra Zeneca, Altana sowie Takeda und halten gelegentliche Vorträge für diese. 
pylori infection in the routine clinical setting. Am J Gastroenterol 1999; 94: 2100-2104

1 Birkner B, Hotz J, Labenz J et al. Kurzgefasste Leitlinie zum Dyspepsie-Management. Dtsch Med Wochenschr 2004; 129 (18): 1032-1034

2 Blaser MJ. Hypothesis: the changing relationship of H.p. and humans: implications for health and disease. J Infect Dis 1999; 179: 1523-1530

3 Brenner $\mathrm{H}$, Arndt V, Stegmaier C et al. Is H.p. infection a necessary condition for noncardia gastric cancer? Am J Epidemiol 2004; 159: 252-258

${ }^{4}$ Chan FK, Chung SC, Suen BY et al. Preventing recurrent upper gastrointestinal bleeding in patients with Helicobacter pylori infection who are taking low-dose aspirin or naproxen. N Engl J Med 2001; 344: 967-973

${ }^{5}$ Chan FK, To KF, Wu JC et al. Eradication of H.p. and risk of peptic ulcers in patients starting long-term treatment with non-steroidal anti-inflammatory drugs: a randomised trial. Lancet 2002; 359: 9-13

${ }^{6}$ Chiba N, Veldhuyzen Van Zanten SJO, Escobedo S et al. Economic evaluation of H.p. eradication in the CADET-Hp randomized controlled trial of H.p. positive primary care patients with uninvestigated dyspepsia. Aliment Pharmacol Ther 2004; 19: 349-358

7 Chiba N, Veldhuyzen Van Zanten SJO, Sinclair P et al. Treating H.p. infection in primary care patients with uninvestigated dyspepsis: the Canadian adult dyspepsia empiric treatment - H.p. positive (CADET-Hp) randomised controlled trial. Brit Med J 2002; 324: 1-7

8 Churin Y, Al-Ghoul L, Kepp O et al. Helicobacter pylori CagA protein targets the c-Met receptor and enhances the motogenic response. J Cell Biol 2003; 28 (161): 249-255

9 Dore MP, Osato MS, Malaty HM et al. Characterization of a culture method to recover Helicobacter pylori from the feces of infected patients. Helicobacter 2000; 5: 165-168

10 El Omar EM, Rabkin CS, Gammon MD et al. Increased risk of noncardia gastric cancer associated with proinflammatory cytokine gene polymorphisms. Gastroenterology 2003; 124: 1193-201

11 Fischbach W, Goebeler-Kolve ME, Dragosics B et al. Long term outcome of patients with gastric marginal zone B cell lymphoma of mucosa associated lymphoid tissue (MALT) following exclusive Helicobacter pylori eradication therapy: experience from a large prospective series. Gut 2004; 53: 34-37

12 Gisbert J, Calvet X, Bujanda L et al. 'Rescue' therapy with rifabutin after multiple Helicobacter pylori treatment failures. Helicobacter 2003; 8: 90-94

13 Glupczynski Y, Megraud F, Lopez-Brea M et al. European multicentre survey of in vitro antimicrobial resistance in Helicobacter pylori. Eur J Clin Microbiol Infect Dis 2001; 20: 820-823

14 Glynn MK, Friedman CR, Gold BD et al. Seroincidence of Helicobacter pylori infection in a cohort of rural Bolivian children: acquisition and analysis of possible risk factors. Clin Infect Dis 2002; 35: 1059-1065

15 Granquist A, Bredberg A, Sveger T et al. A longitudinal cohort study on the prevalence of Helicobacter pylori antibodies in Swedish children and adolescents. Acta Paediatr 2002; 91: 636-640

${ }^{16}$ Grimm W, Fischbach W. Helicobacter pylori infection in children and juveniles: an epidemiological study on prevalence, socio-economic factors and symptoms. Dtsch Med Wochenschr 2003; 128: 1878-1883

17 Heuberger F, Pantoflickowa D, Gassner M et al. H.p. infection in Swiss adolescents. Prevalence and risk factors. Eur J Gastroenterol Hepatol 2003; 15: $179-183$

18 Huang J, Hunt R. The importance of clarithromycin dose in the management of Helicobacter pylori infection: a meta-analysis of triple therapies with a proton pump inhibitor, clarithromycin and amoxicillin or metronidazole. Aliment Pharmacol Ther 1999; 13: 857-64

19 Kist M, Glocker E. Helicobacter pylori Infektionen: ResiNet - eine bundesweite Sentinelstudie zur Resistenzentwicklung. Epidemiol Bull 2003; 47: 389-391

20 Klotz U, Schwab M, Treiber G. CYP2C19 Polymorphism and Proton Pump Inhibitors. Basic Clin Pharmacol Toxicol 2004; 95: 2-8

${ }^{21}$ Kuipers EJ, Nelis GF, Klinkenberg-Knol EC et al. Cure of Helicobacter pylori infection in patients with reflux oesophagitis treated with long term omeprazole reverses gastritis without exacerbation of reflux disease: results of a randomised controlled trial. Gut 2004; 53: 12-20

22 Labenz J, Blum AL, Bolten WW et al. Primary prevention of diclofenac associated ulcers and dyspepsia by omeprazole or triple therapy in H.p. positive patients: a randomised, double blind, placebo controlled, clinical trial. Gut 2002; 51: 329-335

${ }^{23}$ Lai KC, Lam SK, Chu KM et al. Lansoprazole for the prevention of recurrences of ulcer complications from long-term low-dose aspirin use. N Engl J Med 2002; 346: 2033-2038

24 Leodolter A, Dominguez-Munoz JE, Von Arnim U et al. Validity of a modified 13C-urea breath test for pre- and posttreatment diagnosis of Helicobacter

25 Leodolter A, Malfertheiner P. Aktuelle diagnostische Methoden zum Nachweis der Helicobacter-pylori-Infektion. Dtsch Med Wochenschr 2001; 126: 207-209

${ }^{26}$ Leodolter A, Wolle K, Malfertheiner P. Current standards in the diagnosis of H. pylori infection. Dig Dis 2001; 19: 116-122

27 Leodolter A, Peitz U, Ebert MP et al. Comparison of two enzyme immunoassays for the assessment of Helicobacter pylori status in stool specimens after eradication therapy. Am J Gastroenterol 2002; 97: 1682-1686

28 Leodolter A, Vaira D, Bazzoli F et al. European multicentre validation trial of two new non-invasive tests for the detection of Helicobacter pylori antibodies: urine-based ELISA and rapid urine test. Aliment Pharmacol Ther 2003; 18: 927-931

29 Leodolter A, Wolle K, Peitz U et al. Evaluation of a near-patient fecal antigen test for the assessment of Helicobacter pylori status. Diagn Microbiol Infect Dis 2004; 48: 145-147

30 Malfertheiner P. H.p. eradication does not exacerbate gastro-oesophageal reflux disease. Gut 2004; 53: 312-313

31 Malfertheiner P, Megraud F, O'Morain C et al. Current concepts in the management of Helicobacter pylori infection - The Maastricht 2-2000 Consensus Report. Aliment Pharmacol Ther 2002; 16: 167-180

32 Malfertheiner P, Mössner J, Fischbach W et al. H.p. eradication is beneficial in the treatment of functional dyspepsia. Aliment Pharmacol Ther 2003; 15: 615-625

33 Malfertheiner P, Peitz U, Treiber G. What constitutes failure for Helicobacter pylori eradication therapy? Can J Gastroenterol 2003; 17 (Suppl B): 53B-57B

34 Manes G, Menchise A, De Nucci C et al. Empirical prescribing for dyspepsia. randomised controlled trial of test and treat versus omeprazole treatment. Brit Med J 2003; 326: 1118

35 Mc Coll KEL, Murray LS, Gillen D et al. Randomised trial of endoscopy with testing for Helicobacter pylori compared with non-invasive H.p. testing alone in the management for dyspepsia. Brit Med J 2002; 324: 999-1002

36 Meining AG, Bayerdorffer E, Stolte M. Helicobacter pylori gastritis of the gastric cancer phenotype in relatives of gastric carcinoma patients. Eur J Gastroenterol Hepatol 1999; 11: 717-720

37 Melo ET, Lopes EP, Almeida JR, Albuquerque HF, Moura IM. Seroprevalence of H. pylori antibodies in medical students and residents in Recife, Brazil. Clin Gastroenterol 2003; 36: 134-8

38 Mitchell HM, Hu P, Chi Y et al. A low rate of reinfection following effective therapy against Helicobacter pylori in a developing nation. Gastroenterology 1998; 114: 256-261

39 Nardone G, Morgner A. Helicobacter pylori and gastric malignancies. Helicobacter 2003; 8,1: 44-52

40 Osato MS, Reddy R, Graham DY. Metronidazole and clarithromycin resistance amongst Helicobacter pylori isolates from a large metropolitan hospital in the United States. Int J Antimicrob Agents 1999; 12: 341-347

41 Peitz U, Leodolter A, Wex T et al. Diagnostics of Helicobacter pylori infection in patients with peptic ulcer bleeding. Z Gastroenterol 2004; 42: 141-146

42 Raymond J, Kalach N, Bergeret M et al. Prevalence of Helicobacter pylori infection in children according to their age. A retrospective study. Arch Pediatr 1998; 5: 617-620

43 Roma-Giannikou E, Karameris A, Balatsos B et al. Intrafamilial spread of Helicobacter pylori: a genetic analysis. Helicobacter 2003; 8: 15-20

44 Rothenbacher D, Brenner H. Burden of H.p. and H.p.-related diseases in developed countries: recent developments and future implications. Microbes Infect 2003; 5 (8): 693-703

45 Schilling D, Demel A, Adamek HE et al. A negative rapid urease test is unreliable for exclusion of Helicobacter pylori infection during acute phase of ulcer bleeding. A prospective case control study. Dig Liver Dis 2003; 35: 217-221

46 Suerbaum S, Michetti P. Helicobacter pylori infection. N Engl J Med 2002; 347: $1175-86$

47 Triantafillidis JK, Gikas A, Hyphantis T et al. Helicobacter pylori infection in hospital workers over a 5-year period. Correlation with demographic and clinical parameters. J Gastroenterol 2002; 37: 1005-1013

48 Uemura N, Okamoto S, Yamamoto S et al. H.p. infection and the development of gastric cancer. N Engl J Med 2001; 345: 784-789

49 Wolle K, Leodolter A, Malfertheiner Pet al. Antibiotic susceptibility of Helicobacter pylori in Germany: stable primary resistance from 1995 to 2000 . J Med Microbiol 2002; 51: 705-9

50 Wong BCY, Lam SK, Wong WM et al. H.p. eradication to prevent gastric cancer in a high-risk region of China. J Amer Med Assoc 2004; 291 (2): 187-194 